\title{
SAÚDE MENTAL DE ESTUDANTES UNIVERSITÁRIOS DURANTE A PANDEMIA DE COVID-19
}

\author{
MENTAL HEALTH OF UNIVERSITY STUDENTS \\ DURING THE COVID-19 PANDEMIC
}

\section{SALUD MENTAL DE LOS ESTUDIANTES UNIVERSITARIOS DURANTE LA PANDEMIA DE COVID-19}

\author{
Vivian Andrade Gundim ${ }^{1}$ \\ Jhonatta Pereira da Encarnação ${ }^{2}$ \\ Flávia Costa Santos ${ }^{3}$ \\ Josenaide Engracia dos Santos ${ }^{4}$ \\ Erika Antunes Vasconcellos ${ }^{5}$ \\ Rozemere Cardoso de Souza
}

Como citar este artigo: Gundim VA, Encarnação JP, Santos FC, Santos JE, Vasconcellos EA, Souza RC. Saúde mental de estudantes universitários durante a pandemia de COVID-19. Rev baiana enferm. 2021;35:e37293.

Objetivo: identificar as evidências disponíveis sobre formas de apresentação do sofrimento psíquico e ações de proteção e promoção da saúde mental em estudantes universitários, durante a pandemia de COVID-19. Método: revisão integrativa de literatura, realizada por etapas: elaboração da pergunta norteadora; estabelecimento de critérios de inclusão e exclusão; definição das informações a serem extraídas; análise crítica dos estudos e dos resultados; apresentação da revisão relacionada ao impacto da pandemia aos estudantes e às ações de proteção e promoção da saúde mental. Resultados: dentre os 7 artigos investigados, publicados em 2020, observaram-se reações emocionais descritas por estresse, ansiedade, luto, raiva e pânico, associadas à preocupação com o atraso das atividades acadêmicas e ao medo de adoecer. Conclusão: é possível afirmar que a pandemia e os aspectos relacionados a ela interferem na vida acadêmica e na saúde dos estudantes e de seus familiares, causando efeitos negativos à sua saúde mental.

Descritores: Estudantes. Estresse psicológico. Coronavírus.

Objective: to identify the available evidence on ways of presenting psychological distress and actions to protect and promote mental bealth in university students during the COVID-19 pandemic. Method: integrative literature review, performed in stages: elaboration of the guiding question; establishment of inclusion and exclusion criteria; definition of the information to be extracted; critical analysis of studies and results; presentation of the review related to the impact of the pandemic on students and mental health protection and promotion actions. Results: among the seven articles investigated, published in 2020, emotional reactions were observed, described by stress, anxiety, grief, anger and panic, associated with concern about the delay of academic activities and fear of falling ill. Conclusion: the + pandemic and its related aspects interfere in the academic life and health of students and their families, causing negative effects on their mental health.

Descriptors: Students. Psychological stress. Coronavirus.

\footnotetext{
Estudante de Enfermagem. Universidade Estadual de Santa Cruz. Ilhéus, Bahia, Brasil. http://orcid.org/0000-0003-3067-35I6.

Estudante de Enfermagem. Universidade Estadual de Santa Cruz. Ilhéus, Bahia, Brasil. http://orcid.org/0000-0002-5331-3866.

Estudante de Enfermagem. Universidade Estadual de Santa Cruz. Ilhéus, Bahia, Brasil. http://orcid.org/0000-0002-9909-6836.

Psicóloga e Terapeuta Ocupacional. Doutora em Ciências Médicas. Professora da Universidade de Brasília. Brasília, Distrito Federal, Brasil. http://orcid. org/0000-0002$77 \mid 2-8470$

Psicóloga. Doutora em Ciências Médicas. Professora da Universidade Estadual de Santa Cruz. Ilhéus, Bahia, Brasil. http://orcid.org/0000-0002-7382-820X.

Enfermeira. Doutora em Enfermagem Psiquiátrica e Saúde Mental. Professora da Universidade Estadual de Santa Cruz. Ilhéus, Bahia, Brasil. rcsouza@uesc.br. http://orcid.org/0000-0003-2705- I556.
} 
Objetivo: identificar la evidencia disponible sobre las formas de presentar angustia psicológica y acciones para proteger y promover la salud mental en los estudiantes universitarios durante la pandemia de COVID-19. Método: revisión integrativa de la literatura, realizada por etapas: elaboración de la pregunta guía; definición de criterios de inclusión y exclusión; definición de la información que se va a extraer; análisis crítico de estudios y resultados; presentación de la revisión relacionada con el impacto de la pandemia en los estudiantes y las acciones de protección y promoción de la salud mental. Resultados: entre los siete artículos investigados, publicados en 2020, se observaron reacciones emocionales descritas por el estrés, la ansiedad, el dolor, la ira y el pánico, asociadas con la preocupación por el retraso de las actividades académicas y el miedo a enfermarse. Conclusión: es posible afirmar que la pandemia $y$ los aspectos relacionados con ella interfieren en la vida académica y la salud de los estudiantes y sus familias, causando efectos negativos en su salud mental.

Descriptores: Estudiantes. Estrés psicológico. Coronavirus.

\section{Introdução}

Em dezembro de 2019, em Wuhan, na China, teve início a epidemia de COVID-19, também reconhecida em diversos países do mundo, caracterizando-se um grande problema de saúde pública $^{(1)}$. Em janeiro de 2020, a Organização Mundial da Saúde (OMS) declarou esse surto como emergência de saúde pública com consequências internacionais ${ }^{(2)}$, e em 11 de março desse mesmo ano, a OMS declarava a COVID-19 como uma pandemia. Esse termo refere-se à distribuição geográfica de uma doença, reconhecendo que existem surtos de COVID-19 em vários países e regiões do mundo. Até o final do mês de maio de 2020 já tinham sido identificados 5.803.416 casos confirmados no mundo, com a notificação de 359.791 óbitos. No Brasil, até o dia 28 de maio de 2020, foram notificados 441.315 casos confirmados e 26.788 óbitos $^{(3)}$.

COVID-19 é o nome dado à infecção causada pelo novo coronavírus (SARS-CoV-2), e apresenta como sintomas febre alta, cansaço e tosse seca. Outros sintomas também apresentados são: dores, congestão nasal, dor de cabeça, conjuntivite, dor de garganta, diarreia, perda de paladar ou olfato e erupção cutânea na pele ou descoloração dos dedos das mãos ou dos pés. Geralmente os sintomas são leves e começam gradualmente $^{(3)}$. Em cerca de $80 \%$ dos casos, a doença evolui beneficamente, sem necessidade de tratamento hospitalar. Entretanto, aqueles que desenvolvem a forma grave da doença, caracterizada como Síndrome Respiratória Aguda Grave (SRAG), têm maiores chances de internações e mortalidade. A letalidade do coronavírus, até então, tem sido majoritariamente associada a pacientes idosos ou à presença de comorbidades, como cardiopatias, diabetes e doenças que afetam o sistema imunológico ${ }^{(4)}$.

Atualmente o momento é de restrição e isolamento social, com medidas de prevenção da infecção, vivenciados por grande parte da população mundial. Como consequência, surge a necessidade de lidar com aspectos que influenciam diretamente na saúde mental da população diante da crise sanitária atual, como medo e pânico, distanciamento de amigos e família, restrição do toque, e vulnerabilidade de pessoas que sofrem violência ${ }^{(5)}$.

As reações psicológicas à crise podem incluir sentimentos de tristeza, medo, opressão, desamparo e raiva, além de dificuldades para se concentrar ou dormir. O medo de entrar em contato com outras pessoas, viajar em transporte público ou entrar em espaços públicos pode aumentar, e algumas pessoas podem ter sintomas físicos, como aumento da frequência cardíaca ou dor no estômago ${ }^{(6)}$.

O isolamento também afeta a educação. A Organização das Nações Unidas para a Educação, a Ciência e a Cultura (UNESCO) estima que o fechamento de instituições de ensino por causa da pandemia está afetando metade dos estudantes no mundo, 890 milhões em 114 países. Alerta-se que o impacto desse isolamento repercutirá no ensino superior global por muito mais tempo depois que o surto for de fato controlado. Nas áreas mais afetadas, as universidades enfrentam a perspectiva de perder um 
semestre ou mais. Com isso, muitos estudantes já enfrentam ansiedade e pânico devido às inúmeras implicações para cursos, tarefas, seminários e defesas de teses adiados ${ }^{(7)}$.

Cerca de metade dos universitários já enfrentam insegurança, medo, sensação de perda e mudanças de humor como consequência da pandemia, comparativamente aos períodos normais $^{(8)}$. Analisados esses fatores, remete-se à relevância de intervenções psicológicas de proteção e promoção da saúde mental, referentes às necessidades emergentes no atual contexto da pandemia ${ }^{(9)}$.

Nesse sentido, tendo em vista as mudanças nos padrões de vida da população mundial e, consequentemente, no cotidiano dos acadêmicos, questionou-se, neste estudo, acerca dos impactos da pandemia da COVID-19 na saúde mental dos estudantes universitários, e quais os mecanismos capazes de minimizar e/ou evitar possíveis sofrimentos e/ou transtornos psíquicos nessa população.

As mídias nacionais e internacionais têm veiculado muitas informações a respeito da pandemia e de seus impactos na população. Essas informações são transmitidas diariamente, acompanhando o fluxo das notícias e, de forma inédita, têm aproximado cientistas dos noticiários, em busca de atualizações e de informações confiáveis ${ }^{(10)}$. Por sua vez, os conhecimentos de base científica relacionados à pandemia da COVID-19 vêm sendo também publicados em nível crescente, conforme a obtenção de resultados de pesquisas e discussões no âmbito científico ${ }^{(11-14)}$.

Com relação à saúde mental, os estudos científicos começaram a ser publicados enfatizando o impacto vivido no momento atual de pandemia, especialmente na vida das pessoas acometidas pela COVID-19 e na vida dos profissionais da saúde que estão na linha de frente ${ }^{(15-17)}$. Estudos na área da Psicologia têm tido como referência outras pesquisas que abordam situações de crise vividas de forma coletiva, dando suporte para o entendimento desse momento de intensa mobilização social $^{(9,18)}$. Ressalta-se, ainda, a abordagem psicossocial do cuidado em saúde mental, com enfoque multidisciplinar e a participação do enfermeiro no manejo e/ou gestão desse suporte às pessoas afetadas e à população em geral ${ }^{(19-20)}$.

Com relação às repercussões na vida acadêmica, os desdobramentos da pandemia também se tornaram foco da atenção, por considerar o risco de infecção de estudantes com participação no enfrentamento da epidemia, a ruptura da rotina de estudos, o adiamento das tarefas e atividades acadêmicas e o atraso no cronograma dos cursos, repercutindo nos planos do futuro profissional e na busca pela independência e estabilidade financeira, em alguns casos, baseada na expectativa de formação universitária. Desde o início da pandemia na China, a universidade caminha em busca de alternativas para o ensino a distância e soluções para os problemas ${ }^{(21-22)}$.

A população acadêmica já vinha sendo objeto de estudos científicos que apontavam para vulnerabilidades relacionadas à saúde mental ${ }^{(23-26)}$. Com a pandemia, instiga-se analisar o que vem sendo debatido nos estudos até o momento, visto que essa análise possibilitará novas medidas preventivas no planejamento de ações inovadoras e de retomada das atividades acadêmicas no período pós-pandemia.

Assim, o presente estudo teve por objetivo identificar as evidências disponíveis sobre formas de apresentação do sofrimento psíquico e ações de proteção e promoção da saúde mental em estudantes universitários, durante a pandemia de COVID-19.

\section{Método}

Trata-se de revisão integrativa de literatura. A revisão integrativa de literatura pode ser considerada como a forma mais ampla de abordagem metodológica, dentre as revisões ${ }^{(27)}$. Esse tipo de revisão permite uma avaliação crítica e a síntese das evidências científicas sobre um tema de investigação, em que o resultado obtido é o estado do conhecimento desse tema, além de permitir a identificação de lacunas e fragilidades que permitem conduzir ao desenvolvimento de futuras investigações ${ }^{(28)}$.

Para esta revisão, as seguintes etapas foram realizadas: elaboração da pergunta norteadora; estabelecimento de critérios de inclusão e 
exclusão dos artigos (seleção da amostra); definição das informações a serem extraídas dos artigos selecionados; análise crítica dos estudos e discussão dos resultados ${ }^{(29)}$; e apresentação da revisão relacionada com o sofrimento psíquico e ações de proteção e promoção da saúde mental em estudantes universitários, durante a pandemia de COVID-19.

Para elaboração da questão norteadora, utilizou-se a estratégia PICOS, um acrônimo no idioma inglês que significa "Paciente, Intervenção, Comparação e Resultados (Outcomes)", sendo elementos fundamentais da questão de pesquisa e da elaboração da pergunta para a busca de evidências na literatura ${ }^{(30)}$.

Desse modo, conferiu-se a $\mathrm{P}$ os estudantes universitários durante a pandemia, a I, a aplicação de ações de proteção e promoção da saúde mental no cuidado em saúde mental, a C, a comparação entre os resultados obtidos, e a $\mathrm{O}$, a presença de evidências na literatura sobre a aplicação das ações de proteção e promoção da saúde mental durante o contexto da pandemia, que resultou na seguinte questão norteadora: Quais são as evidências encontradas na literatura sobre espaços e/ou ações de proteção e promoção da saúde mental que estão disponíveis para os estudantes universitários durante a pandemia de COVID-19?

Posteriormente, foram estabelecidas e consultadas as palavras-chave na plataforma de Descritores em Ciências da Saúde (DeCS), a saber: Estudantes, Estresse psicológico e Coronavírus. Em seguida, procedeu-se as etapas de busca e seleção de artigos (Fluxograma 1). A coleta de dados foi realizada entre maio e 3 de junho de 2020 .

Fluxograma 1 - Amostragem da revisão integrativa

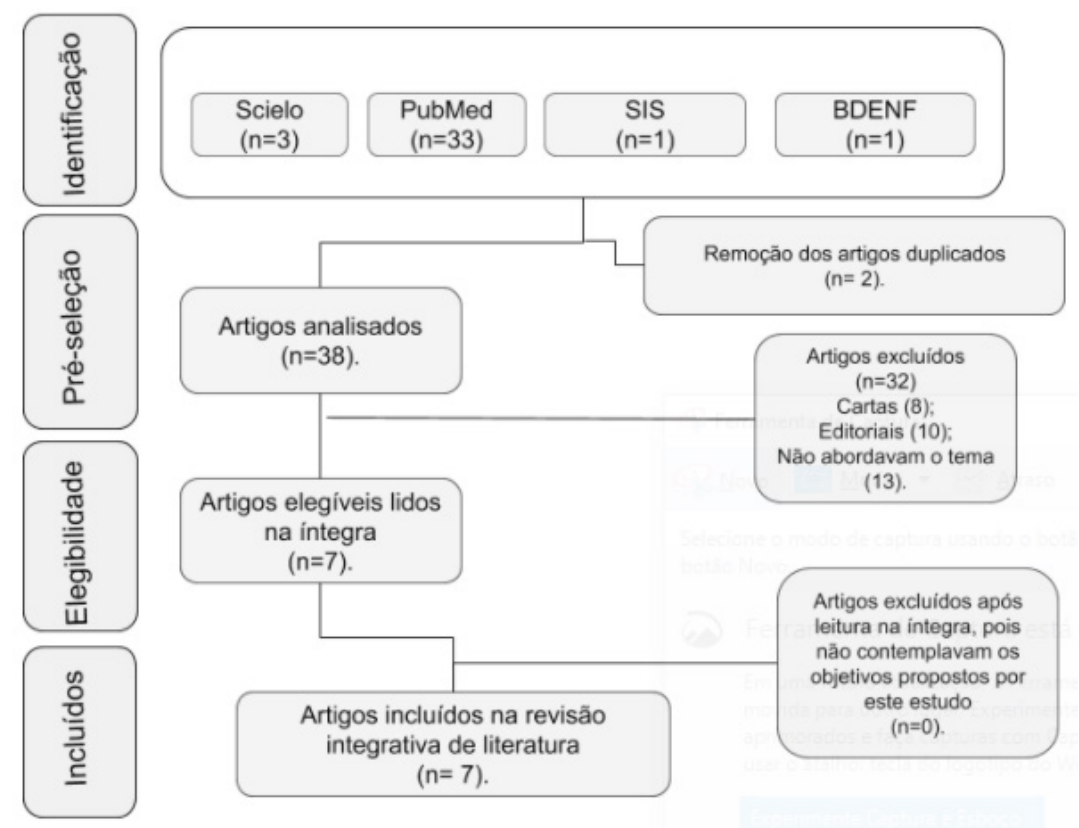

Fonte: Elaboração própria.

Fez-sebuscadeartigosnasseguintesbasesdedados: SCIELO (3), PUBMED (33), LILACS (0), BDENF (1), SIS (Scientific Indexing Services) (1). Foram identificadas 38 publicações elegíveis para a inclusão no trabalho. Após leitura dos resumos e leitura integral dos artigos, quando as informações contidas no resumo não eram suficientes, foram selecionados 7 artigos, que atenderam aos seguintes critérios de inclusão: artigos completos disponíveis eletronicamente de forma gratuita, que apresentavam a temática de estudo como assunto central do trabalho ou com conteúdo relevantes ao tema, em português, espanhol ou inglês. Constituíram critérios de exclusão: cartas ao editor, editoriais, artigos em duplicidades, ou trabalhos que não contemplaram os objetivos do estudo. 
Posteriormente à seleção dos mecanismos de busca e dos critérios de inclusão/exclusão, foram selecionados os aspectos das publicações a serem analisados: título, periódico, ano de publicação, autores, objetivos, características e principais resultados do estudo. Para a análise, fez-se leitura exaustiva dos materiais e utilizou-se um instrumento de descrição desses conteúdos de forma sucinta, o que possibilitou a definição de elegibilidade dos dados para a elaboração da revisão, de forma a atingir seus objetivos ${ }^{(31)}$.

Desse modo, os resultados estão descritos em categorias temáticas, entrelaçando-os à literatura investigada e à interpretação dos pesquisadores, dando evidências à diversidade e aos temas recorrentes nos artigos, e também à sua aplicabilidade para a Enfermagem e para o campo de atenção psicossocial.
Descreveu-se as publicações de forma sucinta para apresentação e discussão dos resultados obtidos, o que possibilitou a definição da elegibilidade dos dados, de forma a atingir seus objetivos. Foram comparados os resultados obtidos individualmente. Em seguida, os resultados foram discutidos e apresentados em duas categorias: impacto da pandemia à saúde mental dos estudantes; mecanismos de proteção e promoção da saúde mental no contexto universitário.

\section{Resultados}

A amostra bibliográfica final foi constituída de sete artigos, publicados no ano de 2020, apresentados no Quadro 1, de acordo com os objetivos deste estudo.

Quadro 1 - Demonstrativo dos artigos selecionados, segundo título, periódico, ano de publicação, autores, principais resultados e característica do estudo.

(continua)

\begin{tabular}{|c|c|c|c|c|c|}
\hline $\begin{array}{c}\text { No de } \\
\text { ordem }\end{array}$ & $\begin{array}{l}\text { Autor e ano/ País } \\
\text { de afiliação dos } \\
\text { autores }\end{array}$ & Periódico & $\begin{array}{c}\text { Contexto/ } \\
\text { Participantes }\end{array}$ & $\begin{array}{l}\text { Tipo de } \\
\text { pesquisa }\end{array}$ & Principais resultados \\
\hline 1 & $\begin{array}{l}\text { Araújo FJO, Lima } \\
\text { LSA, Cidade PIM, } \\
\text { Nobre CB, } \\
\text { Rolim Neto ML. } \\
\text { 2020/ } \text { Brasil }^{(7)}\end{array}$ & $\begin{array}{l}\text { Psychiatry } \\
\text { Research }\end{array}$ & $\begin{array}{l}\text { Os autores } \\
\text { não } \\
\text { especificam } \\
\text { os critérios } \\
\text { utilizados na } \\
\text { metodologia }\end{array}$ & $\begin{array}{l}\text { Revisão de } \\
\text { literatura }\end{array}$ & $\begin{array}{l}\text { O impacto psicológico } \\
\text { causado pela pandemia } \\
\text { de COVID-19 tem sido um } \\
\text { disruptor crítico, criando } \\
\text { ansiedade e depressão, } \\
\text { exacerbadas por incertezas } \\
\text { e intensificação do fluxo } \\
\text { de informações. Com } \\
\text { isso, evidencia-se a } \\
\text { necessidade das instituições } \\
\text { considerarem medidas } \\
\text { preventivas em larga escala } \\
\text { para manter estudantes e } \\
\text { professores saudáveis, física } \\
\text { e psicologicamente, bem } \\
\text { como para criar planos } \\
\text { para quando as aulas } \\
\text { retornarem e as infecções se } \\
\text { manifestarem nos campus. }\end{array}$ \\
\hline 2 & $\begin{array}{l}\text { Maia BR, Dias PC, } \\
2020 / \text { Brasil }^{(8)}\end{array}$ & $\begin{array}{l}\text { Estudos de } \\
\text { Psicologia }\end{array}$ & $\begin{array}{l}619 \\
\text { estudantes } \\
\text { de } 2 \\
\text { universidades } \\
\text { portuguesas } \\
\text { em } 2 \\
\text { períodos de } \\
\text { tempo. }\end{array}$ & $\begin{array}{l}\text { Estudo } \\
\text { descritivo } \\
\text { transversal }\end{array}$ & $\begin{array}{l}\text { Verificou-se aumento } \\
\text { significativo de perturbação } \\
\text { psicológica (ansiedade, } \\
\text { depressão e estresse) entre } \\
\text { os estudantes universitários } \\
\text { no período pandêmico, } \\
\text { comparativamente a } \\
\text { períodos normais. Os } \\
\text { autores não abordam ações } \\
\text { e métodos de proteção e } \\
\text { promoção da saúde mental. }\end{array}$ \\
\hline
\end{tabular}


Quadro 1 - Demonstrativo dos artigos selecionados, segundo título, periódico, ano de publicação, autores, principais resultados e característica do estudo.

(continuação)

\begin{tabular}{|c|c|c|c|c|c|}
\hline $\begin{array}{c}\text { No de } \\
\text { ordem }\end{array}$ & $\begin{array}{c}\text { Autor e ano/ País } \\
\text { de afiliação dos } \\
\text { autores }\end{array}$ & Periódico & $\begin{array}{c}\text { Contexto/ } \\
\text { Participantes }\end{array}$ & $\begin{array}{l}\text { Tipo de } \\
\text { pesquisa }\end{array}$ & Principais resultados \\
\hline 3 & $\begin{array}{l}\text { Schmidt B, } \\
\text { Crepald MA, } \\
\text { Bolze DAS, } \\
\text { Neiva-Silva L, } \\
\text { Demenech LM. } \\
\text { 2020/ } \text { Brasil }^{(9)}\end{array}$ & $\begin{array}{l}\text { Estudos de } \\
\text { Psicologia }\end{array}$ & $\begin{array}{l}53 \text { artigos } \\
\text { envolvendo } \\
\text { a população } \\
\text { geral e } \\
\text { estudantes, } \\
\text { encontrados } \\
\text { nas bases de } \\
\text { dados } \\
\text { ScienceDirect, } \\
\text { PubMed, } \\
\text { SciELO } \\
\text { e Scholar } \\
\text { Google. }\end{array}$ & $\begin{array}{l}\text { Revisão de } \\
\text { literatura }\end{array}$ & $\begin{array}{l}\text { Estudos têm sugerido que o } \\
\text { medo de ser infectado por } \\
\text { um vírus potencialmente } \\
\text { fatal, de rápida disseminação, } \\
\text { cujas origens, natureza } \\
\text { e curso ainda são pouco } \\
\text { conhecidos, acaba por afetar } \\
\text { o bem-estar psicológico de } \\
\text { muitas pessoas. Ser mulher, } \\
\text { estudante e apresentar } \\
\text { sintomas físicos ligados à } \\
\text { COVID-19, ou problemas de } \\
\text { saúde prévios, foram fatores } \\
\text { significativamente associados } \\
\text { a maiores níveis de ansiedade, } \\
\text { depressão e estresse. }\end{array}$ \\
\hline 4 & $\begin{array}{l}\text { Wang C, Pan R, } \\
\text { Wan X, Tan Y, } \\
\text { Xu L,Ho CS, } \\
\text { et al. } \\
\text { 2020/ China e } \\
\text { Sibéria }^{(15)}\end{array}$ & $\begin{array}{l}\text { International } \\
\text { Journal of } \\
\text { Environmental } \\
\text { Research and } \\
\text { Public Health }\end{array}$ & $\begin{array}{l}1304 \\
\text { estudantes } \\
\text { universitários } \\
\text { que vivem } \\
\text { na China } \\
\text { continental } \\
\text { durante a } \\
\text { epidemia de } \\
\text { covid-19 }\end{array}$ & $\begin{array}{l}\text { Estudo } \\
\text { descritivo } \\
\text { transversal }\end{array}$ & $\begin{array}{l}\text { A maioria dos entrevistados } \\
\text { classificou o impacto } \\
\text { psicológico causado pela } \\
\text { pandemia como moderado } \\
\text { ou grave. A incerteza } \\
\text { e o potencial impacto } \\
\text { negativo na progressão } \\
\text { acadêmica podem ter um } \\
\text { efeito adverso na saúde } \\
\text { mental dos estudantes. O } \\
\text { sexo feminino, o status de } \\
\text { estudante e os sintomas } \\
\text { físicos específicos foram } \\
\text { associados a maior impacto } \\
\text { psicológico do surto e } \\
\text { a níveis mais altos de } \\
\text { estresse, ansiedade e } \\
\text { depressão. Medidas de } \\
\text { apoio relatadas incluem: } \\
\text { identificação de grupos de } \\
\text { alto risco para intervenções } \\
\text { psicológicas precoces, } \\
\text { fornecimento de recursos } \\
\text { para apoio psicológico, } \\
\text { desenvolvimento de } \\
\text { redes de suporte on- } \\
\text { line, portais e aplicativos } \\
\text { baseados na Web para } \\
\text { ministrar palestras ou } \\
\text { outras atividades de } \\
\text { ensino e o fornecimento } \\
\text { de informações precisas e } \\
\text { baseadas em evidências em } \\
\text { formato diagramático. }\end{array}$ \\
\hline
\end{tabular}


Quadro 1 - Demonstrativo dos artigos selecionados, segundo título, periódico, ano de publicação, autores, principais resultados e característica do estudo.

(continuação)

\begin{tabular}{|c|c|c|c|c|c|}
\hline $\begin{array}{c}\text { No de } \\
\text { ordem }\end{array}$ & $\begin{array}{c}\text { Autor e ano/ País } \\
\text { de afiliação dos } \\
\text { autores }\end{array}$ & Periódico & $\begin{array}{c}\text { Contexto/ } \\
\text { Participantes }\end{array}$ & $\begin{array}{l}\text { Tipo de } \\
\text { pesquisa }\end{array}$ & Principais resultados \\
\hline 5 & $\begin{array}{l}\text { Teixeira MR, } \\
\text { Dahl CM. } \\
\text { 2020/ Brasil } \\
\end{array}$ & $\begin{array}{l}\text { Revista } \\
\text { Interinstitucional } \\
\text { Brasileira } \\
\text { de Terapia } \\
\text { Ocupacional }\end{array}$ & $\begin{array}{l}\text { Realizado } \\
\text { cruzamento } \\
\text { de dados } \\
\text { contidos na } \\
\text { literatura com } \\
\text { os principais } \\
\text { jornais } \\
\text { internacionais } \\
\text { (CNN, ABC } \\
\text { NEWS, } \\
\text { CNBC, Inside } \\
\text { Higher, The } \\
\text { Hechinger, } \\
\text { The World } \\
\text { University } \\
\text { Rankings, The } \\
\text { Guardian e } \\
\text { Lifestyle). }\end{array}$ & $\begin{array}{l}\text { Revisão de } \\
\text { literatura }\end{array}$ & $\begin{array}{l}\text { Foram observados entre } \\
\text { os estudantes sentimentos } \\
\text { de medo, estresse, } \\
\text { ansiedade, luto, culpa, } \\
\text { raiva e desamparo diante } \\
\text { da incerteza, identificados } \\
\text { como respostas emocionais } \\
\text { comuns em contextos de } \\
\text { emergência. Identificaram- } \\
\text {-se ações de redução } \\
\text { desses impactos, tais como: } \\
\text { organização de uma rotina } \\
\text { de atividades de estudos } \\
\text { de seu interesse, encontros } \\
\text { virtuais para realização } \\
\text { de práticas integrativas e } \\
\text { complementares, atividades } \\
\text { acadêmicas e orientações } \\
\text { de forma remota e apoio } \\
\text { psicossocial, grupos } \\
\text { de apoio, e busca por } \\
\text { profissionais de forma } \\
\text { virtual. }\end{array}$ \\
\hline 6 & $\begin{array}{l}\text { Yuan S, Liao Z, } \\
\text { Huang H, } \\
\text { Jiang B, Zhang X, } \\
\text { Wang Y, Zhao M. } \\
\text { 2020/ China } \\
\text { (33) }\end{array}$ & $\begin{array}{l}\text { International } \\
\text { Medical } \\
\text { Journal of } \\
\text { Experimental } \\
\text { and Clinical } \\
\text { Research }\end{array}$ & $\begin{array}{l}\text { População } \\
\text { da província } \\
\text { chinesa de } \\
\text { Hubei e de } \\
\text { províncias não } \\
\text { endêmicas }\end{array}$ & $\begin{array}{l}\text { Estudo } \\
\text { descritivo } \\
\text { transversal }\end{array}$ & $\begin{array}{l}\text { Da população do estudo, } \\
65,92 \% \text { eram estudantes } \\
\text { universitários. A maioria } \\
\text { dos participantes do } \\
\text { estudo, duas semanas } \\
\text { após o início da } \\
\text { pandemia, relatou que } \\
\text { seu estado emocional } \\
\text { permaneceu inalterado } \\
\text { durante o período de } \\
\text { estudo de duas semanas. } \\
\text { Os autores ressaltam } \\
\text { a importância do } \\
\text { desenvolvimento de ações } \\
\text { que minimizem o impacto } \\
\text { à saúde mental, para } \\
\text { aqueles que manifestaram } \\
\text { alterações. }\end{array}$ \\
\hline
\end{tabular}


Quadro 1 - Demonstrativo dos artigos selecionados, segundo título, periódico, ano de publicação, autores, principais resultados e característica do estudo.

(conclusão)

\begin{tabular}{|c|c|c|c|c|c|}
\hline $\begin{array}{l}\text { No de } \\
\text { ordem }\end{array}$ & $\begin{array}{l}\text { Autor e ano/ País } \\
\text { de afiliação dos } \\
\text { autores }\end{array}$ & Periódico & $\begin{array}{c}\text { Contexto/ } \\
\text { Participantes }\end{array}$ & $\begin{array}{l}\text { Tipo de } \\
\text { pesquisa }\end{array}$ & Principais resultados \\
\hline 7 & $\begin{array}{l}\text { Cao W, Fang Z, } \\
\text { Hou G, Han M, Xu } \\
\text { X, Dong J, Zheng } \\
\text { J. } \\
\text { 2020/ China } \\
\end{array}$ & $\begin{array}{l}\text { Psychiatry } \\
\text { research }\end{array}$ & $\begin{array}{l}7143 \\
\text { Graduandos } \\
\text { da Faculdade } \\
\text { de Medicina } \\
\text { de Changzhi. }\end{array}$ & $\begin{array}{l}\text { Estudo } \\
\text { descritivo } \\
\text { transversal }\end{array}$ & $\begin{array}{l}\text { O estudo mostra que a } \\
\text { saúde mental de estudantes } \\
\text { universitários foi afetada } \\
\text { em graus variados durante } \\
\text { o surto. A pesquisa } \\
\text { indicou que } 24,9 \% \text { dos } \\
\text { estudantes universitários } \\
\text { estavam sofrendo de } \\
\text { ansiedade devido ao surto } \\
\text { de COVID-19 associados a } \\
\text { fatores como: estabilidade } \\
\text { da renda familiar, pressão } \\
\text { psicológica e econômica, } \\
\text { preocupação com atrasos } \\
\text { acadêmicos e a influência } \\
\text { da epidemia na vida diária, } \\
\text { preocupação com estudos } \\
\text { e no emprego futuro, } \\
\text { ausência de comunicação } \\
\text { interpessoal e notícias } \\
\text { falsas. Foi indicado que } \\
\text { o número crescente de } \\
\text { pacientes e casos suspeitos, } \\
\text { bem como o número } \\
\text { crescente de províncias e } \\
\text { países afetados pelo surto, } \\
\text { suscitou preocupação } \\
\text { pública, o que aumentou a } \\
\text { ansiedade. }\end{array}$ \\
\hline
\end{tabular}

Fonte: Elaboração própria.

Os artigos analisados foram em sua maioria filiados à China (3) e ao Brasil (3); os demais ocorreram em Portugal (1). Quanto ao periódico de publicação dos artigos, 2 artigos foram publicados na revista Estudos de Psicologia, e os demais foram distribuídos entre as revistas: Psychiatry Research (2), Revista Interinstitucional Brasileira de Terapia Ocupacional (1), International Journal of Environmental Research and Public Health (1) e International Medical Journal of Experimental and Clinical Research (1).

A maioria dos estudos tratava-se de pesquisas descritivas transversais (4), e o restante utilizou-se do método revisão de literatura (3). Os estudos, em sua maioria, tiveram como população estudantes universitários (4), e em 3 dos estudos, a população em geral, com especificações acerca da saúde mental de estudantes. Em 2 dos estudos, os participantes foram entrevistados antes e durante o período pandêmico, realizando descrição comparativa com relação aos períodos.

Foram utilizadas diferentes escalas para avaliação dos impactos psíquicos causados aos estudantes, dentre elas: Escala de depressão, ansiedade e estresse (EADS-21) ${ }^{(8,15)}$, Escala de transtorno de ansiedade generalizada de 7 itens (GAD-7) $)^{(34)}$, Self-reporting Questionnaire (SRQ-20) em associação com o Índice de Qualidade do Sono de Pittsburgh (PSQI) (estudo 6). Os estudos de 1, 3 e 5 não utilizaram escalas por não se tratarem de estudos descritivos ${ }^{(7,9,32)}$. 
Impacto da pandemia à saúde mental dos estudantes

Compreende-se que no decorrer do período pandêmico foram observadas alterações no bem-estar psíquico de estudantes universitários $^{(8)}$. Salienta-se que certas medidas impostas à população de forma inesperada resultaram em uma reorganização social abrupta, logo, uma das diversas consequências mostra-se refletida na saúde mental da população em geral e de estudantes universitários ${ }^{(9)}$.

Estudo realizado na China revela que diante do cenário de calamidade de saúde pública é normal que as pessoas sintam medo de adoecer ou morrer, e muito provavelmente apresentarão sentimentos de desamparo e estigma com as paralisações de atividades, comércio, escolas, transportes, fazendo com que as emoções negativas tendam a ser agravadas. Estudo abordando os fatores associados ao sofrimento psíquico dos estudantes descreve que $70 \%$ estava preocupado com a possibilidade de seus familiares contraírem a COVID-19 ${ }^{(15)}$. Ainda é possível observar outras reações emocionais, tais como: estresse, ansiedade, luto, culpa e raiva ${ }^{(32)}$.

Quando comparado o período normal ao pandêmico, foram encontradas diferenças estatisticamente significativas nos níveis de ansiedade, depressão e estresse entre os estudantes ${ }^{(14)}$. Associa-se que as medidas rigorosas de isolamento utilizadas como método de prevenção do vírus ocasionaram atrasos no início das aulas de escolas, faculdades e universidades em toda a China, impactando a saúde mental de estudantes universitários. As preocupações com aspectos econômicos, atrasos acadêmicos e a influência da epidemia nas atividades diárias, bem como a escassez de máscaras e as informações e notícias falsas podem ser apontadas como causas do sofrimento psíquico pelo qual esses estudantes estão sendo acometidos ${ }^{(33)}$.

Há relatos de que respostas incertas por parte das universidades sobre a criação de prováveis estratégias relacionadas à retomada das atividades podem causar o sentimento de incerteza e ansiedade nos estudantes, à medida que não se sabe o efeito sobre seus estudos e o reflexo que isso implicará em seu futuro ${ }^{(7,33)}$. Estão associados também outros fatores, como as limitações do acesso aos recursos virtuais, medida que está sendo frequentemente utilizada para dar continuidade às atividades acadêmicas, bem como as mudanças abruptas das rotinas e o temor pela vida dos entes queridos ${ }^{(32)}$.

Apesar das medidas de restrições sociais não serem algo novo, as suas consequências na vida dos estudantes podem causar dor psicológica em diferentes níveis. Estudo descreve que cerca de $15,1 \%$ dos estudantes consideraram sentir-se significativamente mais preocupados durante o período pandêmico quando em referência ao período normal, e 38,5\% declarou "sentir-se com medo". Outras formas de apresentação do sofrimento psíquico apresentadas foram: sentimentos de falta de conforto, preocupação, inquietação, sentimento de não saber o que fazer, sentir que a vida não tinha sentido ou sentir-se quase a entrar em pânico ${ }^{(7)}$.

Ao avaliar o impacto emocional sobre estudantes universitários chineses, obteve-se que a saúde mental foi afetada em graus variados durante o surto. Dos estudantes entrevistados, a pesquisa indicou que $24,9 \%$ estavam sofrendo de ansiedade devido ao surto de COVID-19; destes, $0,9 \%$ sofriam de ansiedade grave, 2,7\% moderada e 21,3\% apresentavam ansiedade leve ${ }^{(15)}$. Em apenas um dos artigos estudados os resultados relatavam melhora na saúde mental dos estudantes ao se comparar o período pandêmico e o período normal ${ }^{(33)}$.

As consequências do sofrimento psíquico para a sociedade em seu conjunto e para os próprios estudantes, tende a perpassar o período pós-pandemia, atingindo dessa forma a sociedade em suas diversas esferas ${ }^{(9)}$. Frente às evidências de que esses efeitos podem prolongar-se ao longo do tempo, ressalta-se a necessidade de traçar estratégias de prevenção ou redução de danos, num trabalho conjunto de diversas instituições públicas, mediante junção de ações de saúde e educação ${ }^{(8)}$.

\section{Mecanismos de proteção e promoção da} saúde mental no contexto universitário

Ressalta-se a importância de que os sistemas de saúde e as autoridades governamentais 
estejam preparados para as transmissões generalizadas de doenças, bem como para todas as consequências possíveis, incluindo os impactos psicológicos a que esses indivíduos estão submetidos diante uma situação de epidemia ${ }^{(15)}$. Frente ao período de emergência de saúde pública, entende-se a necessidade de identificar medidas de prevenção e promoção de saúde mental desses estudantes ${ }^{(7)}$.

Autores identificaram que evitar o compartilhamento de utensílios, higienização das mãos e uso de máscaras, foram associados a níveis mais baixos de impacto psicológico, depressão, ansiedade e estresse ${ }^{(15)}$. Tal qual a divulgação de dados precisos e reais sobre a situação da doença, evitar a exposição excessiva a noticiários de televisão, a busca por veracidade dos fatos noticiados ou descobertos, também se revelam redutores desses níveis ${ }^{(9)}$.

Estuda-se a aplicação de questionários específicos para os cursos de graduação e pós-graduação como mecanismo para identificar as diferentes formas de sofrimento, classificá-las de acordo com sua gravidade e risco, para então poder traçar um plano de cuidados para minimizar ou cessar esse sofrimento no ambiente acadêmico. A adaptação de atividades remotas de acordo com a agenda do estudante seria uma forma de minimizar os atrasos no calendário acadêmico, e a criação de encontros virtuais para as práticas integrativas e complementares, um meio de minimização desse estresse, e de promoção de relaxamento ${ }^{(32)}$.

Os jovens são mais receptivos às atualizações on-line e às redes de smartphones. Esse é um fator facilitador para enfrentamento dos desafios do ensino superior. Uma estratégia possível seria o fornecimento de atividades educativas e intervenções psicológicas por meio desses meios, para redução dos impactos à saúde mental dos estudantes, além do desenvolvimento de portais e aplicativos on-line para ministrar palestras ou atividades de ensino ${ }^{(15)}$. Também se faz importante a elaboração de atendimentos psicoeducativos utilizando-se cartilhas e materiais informativos, e meios para o desenvolvimento da escuta psicológica para o alívio das emoções, adaptando tais materiais às condições do público-alvo, por meio da linguagem e da forma de divulgação ${ }^{(9)}$.

Não obstante o retorno das atividades acadêmicas ser um mecanismo de prevenção do sofrimento psíquico, há a necessidade de não sobrecarregar o estudante nesse período. Formas de adaptação para atender as diversas realidades que compõem o ambiente acadêmico são relevantes, por exemplo, o atendimento por ligação telefônica para indivíduos que não possuem acesso à Internet, criação de conteúdos que consumam poucos Gb (Gigabit), questionários com o intuito de conhecer a individualidade do estudante para melhor aproveitamento das atividades propostas, bem como o desenvolvimento de ações de apoio social ${ }^{(32)}$. O apoio social reduz a pressão psicológica durante as epidemias e torna-se eficaz e necessário durante emergências de saúde pública ${ }^{(15)}$.

Salienta-se a importância da participação dos diferentes grupos e atores sociais na elaboração do levantamento das questões psicossociais ${ }^{(16)}$. Além disso, as autoridades de saúde precisam identificar as necessidades psicológicas desses estudantes durante a pandemia. Os profissionais de saúde também têm o dever de fornecer recursos para apoio psicológico e intervenções, especialmente durante uma hospitalização ${ }^{(34)}$. Ressalta-se também o importante papel dos profissionais não especializados que pretende mitigar o sofrimento emocional e diminuir os possíveis agravantes ${ }^{(32)}$.

\section{Discussão}

O sofrimento psíquico entre universitários nos estudos investigados apresenta-se na forma de estresse, sentimento de incapacidade frente ao isolamento, incômodo com a situação de constante vigília, preocupação com os cuidados preventivos, medo de perder parentes e amigos, preocupação com a situação econômica do país, sinais de ansiedade e depressão, além de dores de cabeça, irritação, autoisolamento, perda da vontade de realizar atividades, angústia e até distúrbios alimentares. 
$\mathrm{Na}$ identificação das possíveis causas de alteração da saúde mental entre os estudantes, são perceptíveis fatores como a quebra da rotina acadêmica, o afastamento de amigos e colegas, a preocupação com o atraso de atividades, consequentemente causando atraso no prosseguimento do curso e na data de formatura, a interrupção de aulas práticas e estágios curriculares, bem como em algumas universidades, a realização de atividades de maneira remota, resultando em preocupações com o acesso à Internet e dificuldades na adaptação ao novo método de aprendizagem.

Não obstante um dos estudos contradizer essas perspectivas ${ }^{(33)}$, outros estudos identificaram que a pandemia da COVID-19 causou alterações no estado emocional e psíquico dos estudantes, sendo associados a fatores, como o isolamento social, o medo frente a possibilidade de adoecimento e morte e o grande fluxo de informações. Entre os estudantes identifica-se que, além dos fatores descritos, o afastamento das atividades acadêmicas tem sido fator crucial causador de sofrimento psíquico.

É imprescindível o uso de medidas que tenham como objetivo minimizar o sofrimento psíquico e seus efeitos negativos em estudantes universitários. Identificam-se diversas estratégias com poder de auxiliar a diminuição dos impactos psicológicos, que vão desde a observação e detecção precoce até a criação de mecanismos que facilitem o acesso desses estudantes às plataformas on-line, para o desenvolvimento de atividades de ensino remotas e apoio psicossocial no contexto da pandemia.

No cotidiano, observa-se que existem certos impasses que dificultam o acesso às atividades remotas por grande parte dos estudantes, tais como: a falta do conhecimento tecnológico relacionado aos meios de comunicação, as condições sociais e econômicas dos estudantes e até mesmo o desânimo em participar das ações propostas. Isso dificulta a criação de estratégias, uma vez que não garante o acesso uniforme e equânime a todos os estudantes.

Além disso, os achados tornam possíveis refletir sobre a implementação das ações de
Enfermagem na atenção aos estudantes universitários. Uma estratégia viável seria a escuta terapêutica dentro do ambiente acadêmico, com o desenvolvimento de um olhar holístico para os discentes, identificando possíveis sofrimentos psíquicos, orientando para a importância do distanciamento social nesse cenário pandêmico, mas garantindo também a continuidade do contato com seu ciclo social, mesmo que virtualmente. É necessário também estimular o autocuidado e a procura por profissionais em situações de agravos. Para a viabilidade dessas ações faz-se importante a busca constante de adaptações nas formas de atendimento, para que seja garantido um atendimento baseado nas necessidades estudantis.

Nos materiais analisados, percebeu-se que apenas um dos artigos utilizou a metodologia de coleta de dados em dois períodos, realizando um comparativo do período pandêmico e o período normal. A maior parte dos estudos realizou a coleta de dados em um período muito curto após o início da pandemia (1 ou 2 semanas), o que faz supor que esses resultados estão aquém da realidade atual, em que já se passou um longo tempo de circulação do vírus e isolamento social. Tal suposição faz crer que há a necessidade da realização de estudos que avaliem os impactos causados ao estado psíquico desses estudantes em longo prazo.

Dentre as dificuldades encontradas para a elaboração deste estudo, inclui-se o fato de que se trata de uma situação recente e emergente, em que ainda há um curto conjunto de artigos referentes à associação da saúde mental de estudantes universitários e a pandemia de COVID-19. Portanto, faz-se necessária a realização de estudos nessa temática, sejam eles longitudinais, descritivos, mas principalmente com ênfase em estudos qualitativos, a fim de valorizar o estudo de aspectos subjetivos relacionados aos impactos causados e às formas de apresentação do sofrimento psíquico nesses indivíduos.

As informações obtidas neste estudo poderão servir como subsídios para estratégias de manejo do estado emocional de universitários. As universidades precisarão pensar formas de minimizar 
os efeitos psicológicos causados pela pandemia, mediante a prestação de assistência psicossocial, como mecanismo para diminuir os impactos ao calendário acadêmico e à própria rotina de atividades, no que diz respeito à circulação de pessoas e do vírus.

\section{Conclusão}

Os artigos abordados neste estudo atenderam ao objetivo proposto, na medida em que foi possível identificar, mediante seus resultados, as formas de apresentação do sofrimento psíquico, bem como os fatores decorrentes da pandemia associados a eles e as possíveis intervenções para minimizá-los ou evitá-los.

Por conseguinte, é possível afirmar que a própria pandemia e seus aspectos que interferem na vida acadêmica e na saúde física dos estudantes e de seus familiares causam efeitos negativos à saúde mental dos universitários e da sociedade de modo geral. Entende-se que é mister, portanto, que medidas de redução, estratégias de prevenção e manejo do sofrimento psíquico sejam adotadas, tanto por parte das autoridades de saúde quanto pelas universidades e profissionais da educação, para minimizar o sofrimento psíquico atual ocorrido durante a pandemia, bem como os efeitos futuros decorrentes dela.

Um estudo aprofundado acerca desses impactos e das formas de apresentação nesses estudantes ainda se faz relevante. Estudos como este refletem a realidade vivida e servem como subsídio para ações de promoção da saúde mental e redução dos efeitos negativos causados por esse período de emergência mundial, porém ressalta-se a importância de que sejam realizados mais estudos nessa temática, principalmente de maneira longitudinal e abordagem qualitativa, além da necessidade de avaliação criteriosa dos danos que podem ocorrer em longo prazo.

\section{Colaborações:}

1 - concepção, projeto, análise e interpretação dos dados: Vivian Andrade Gundim, Jhonatta Pereira da Encarnação, Flávia Costa Santos e Rozemere Cardoso de Souza;
2 - redação do artigo e revisão crítica relevante do conteúdo intelectual: Vivian Andrade Gundim, Jhonatta Pereira da Encarnação, Flávia Costa Santos, Josenaide Engracia dos Santos, Erika Antunes Vasconcellos e Rozemere Cardoso de Souza;

3 - aprovação final da versão a ser publicada: Rozemere Cardoso de Souza.

\section{Referências}

1. Phelan AL, Katz R, Gostin LO. The Novel Coronavirus Originating in Wuhan, China: Challenges for Global Health Governance. Jama. 2020;323(8):709-10. DOI: 10.1001/jama.2020.1097

2. Meng L, Hua F, Bian Z. Coronavirus Disease 2019 (COVID-19): Emerging and Future Challenges for Dental and Oral Medicine. J Dent Res. 2020;99(5):481-7. DOI: 10.1177/0022034520914246

3. Organização Mundial de Saúde. Doença de coronavírus 2019 (COVID-19): relatório de situação-36 [Internet]. Genebra (CHE); 2020 [cited 2020 May 29]. Available from: https://www.paho. org/pt/covid19

4. Lana RM, Coelho FC, Gomes MFDC, Cruz OG, Bastos LS, Villela DAM, et al. Emergência do novo coronavírus (SARS-CoV-2) e o papel de uma vigilância nacional em saúde oportuna e efetiva. Cad Saúde Pública. 2020;36(3):e00019620. DOI: http:// dx.doi.org/10.1590/0102-311x00019620

5. Ferreira MG, Barbosa EI. Antagonismo do isolamento: o distanciamento que protege e vulnerabiliza frente ao contexto de pandemia [Internet]. Health Residencies Journal. 2020 [cited 2020 May 16];1(3):1-5. Available from: https:// escsresidencias.emnuvens.com.br/hrj/article/ view/36

6. Freitas F. Coronavírus, saúde mental e o que levar em conta no isolamento [Internet]. Rio de Janeiro (RJ): Fundação Oswaldo Cruz; 2020 [cited 2020 Jun 7]. Available from: https://cee.fiocruz. $\mathrm{br} /$ ?q=Coronavirus-saude-mental-e-o-que-levarem-conta-no-isolamento

7. Araújo FJO, Lima LSA, Cidade PIM, Nobre CB, Rolim Neto ML. Impact of Sars-Cov-2 And Its Reverberation in Global Higher Education and Mental Health. Psychiatry Res. 2020; 288:112977. DOI: $10.1016 /$ j.psychres.2020.112977

8. Maia BR, Dias PC. Ansiedade, depressão e estresse em estudantes universitários: o 
impacto da COVID-19. Estud psicol (Campinas). 2020;37:e200067. DOI: http://dx.doi. org/10.1590/1982-0275202037e200067

9. Schmidt B, Crepald MA, Bolze DAS, Neiva-Silva L, Demenech LM. Saúde mental e intervenções psicológicas diante da pandemia do novo coronavírus (COVID-19). Estud psicol (Campinas). 2020;37:e200063. DOI: https://dx.doi. org/10.1590/1982-0275202037e200063

10. Xavier AAO, Barata G, Tercic LS, Hafiz M. Covid-19 aproxima cientistas da mídia de maneira inédita [Internet]. Campinas: Unicamp; 2020 [cited 2020 Jun 10]. Available from: https://www.unicamp. br/unicamp/coronavirus/covid-19-aproximacientistas-da-midia-de-maneira-inedita

11. Li H, Liu SM, Yu XH, Tang SL, Tang CK. Coronavirus disease 2019 (COVID-19): current status and future perspectives. Int $\mathrm{J}$ Antimicrob Agents. 2020;55(5). DOI: https://doi.org/10.1016/j. ijantimicag.2020.105951

12. Arshad Ali S, Baloch M, Ahmed N, Arshad Ali A, Iqbal A. The outbreak of Coronavirus Disease 2019 (COVID-19) - An emerging global health threat. J Infect Public Health. 2020 Apr;13(4):644-6. DOI: https://doi.org/10.1016/j.jiph.2020.02.033

13. Freitas ARR, Napimoga M, Donalisio MR. Análise da gravidade da pandemia de Covid-19. Epidemiol Serv Saúde. 2020;29(2):e2020119. DOI: https:// doi.org/10.5123/s1679-49742020000200008

14. Estrela FM, Cruz MA, Gomes NP, Oliveira MAS, Santos RS, Magalhães JRF, et al. Covid-19 e doenças crônicas: impactos e desdobramentos frente à pandemia. Rev baiana enferm. 2020;34:e36559. DOI: $10.18471 /$ rbe.v34.36559

15. Wang C, Pan R, Wan X, Tan Y, Xu L, Ho CS, et al. Immediate psychological responses and associated factors during the initial stage of the 2019 coronavirus disease (COVID-19) epidemic among the general population in China. Int J Environ Res Public Health. 2020;17(5):1729. DOI: 10.3390/ijerph17051729

16. Organização Pan-Americana da Saúde. Considerações psicossociais e de saúde mental durante o surto de COVID-19, 18 de março de 2020. Washington, D. C. (EUA); 2020 [cited 2020 Mar 28]. Available from: https://iris.paho.org/ handle/10665.2/51996

17. Miyazaki MCOS, Soares MRZ. Estresse em profissionais da saúde que atendem pacientes com COVID-19. Ribeirão Preto: Sociedade Brasileira de
Psicologia; 2020 [cited 2020 Jun 11]. Available from: https://www.sbponline.org.br/2020/03/grupo-detrabalho-gt-de-enfrentamento-da-pandemia-sbpcovid-19

18. Enumo SRF, Weide JN, Vicentinni ECC, Araújo MF, Machado WL. Enfrentando o estresse em tempos de pandemia: proposição de uma Cartilha. Estud psicol (Campinas). 2020;37:e200065. DOI: https:// doi.org/10.1590/1982-0275202037e200065

19. Crispim D, Silva MJPS, Cedotti W, Câmara M, Ananda GS. Visitas virtuais durante a pandemia do Covid-19 [Internet]. Belo Horizonte: Associação Médica de Minas Gerais; 2020 [cited 2020 Apr 20]. Available from: https://ammg.org.br/wp-content/ uploads/Visitas-virtuais-COVID-19.pdf

20. Cruz N, Souza E, Sampaio C, Santos A, Chaves S, Hora R, et al. Apoio psicossocial em tempos de COVID-19: experiências de novas estratégias de gestão e ajuda mútua no sul da Bahia, Brasil. APS em Revista. 2020;2(2):97-105. DOI: https:// doi.org/10.14295/aps.v2i2.94

21. Wang C, Cheng Z, Yue XG, McAleer M. Risk Management of COVID-19 by Universities in China. J Risk Financial Manag. 2020;13(2):36. DOI:10.3390/jrfm13020036

22. Senhoras EM. Coronavírus e educação: análise dos impactos assimétricos. Boletim de Conjuntura (BOCA). 2020:2(5):128-36. DOI: http://dx.doi. org/10.5281/zenodo.3828085

23. Arino DO, Bardagi MP. Relação entre Fatores Acadêmicos e a Saúde Mental de Estudantes Universitários. Psicol pesq. 2018;12(3):44-52. DOI: http://dx.doi.org/10.24879/2018001200300544

24. Santos RR, Greco PBT, Prestes FC, Kirchhof RS, Magnago TSBS, Oliveira MA. Síntomas de Transtornos Psíquicos Menores en estudiantes de enfermería. Rev baiana enferm. 2016;30(3):1-14. DOI: $10.18471 /$ rbe.v30i3.16060

25. Pereira A, Cardoso F. Ideação suicida na população universitária: Uma revisão da literatura. Rev E-Psi [Internet]. 2015 [cited 2020 May 10];5(2):16-34. Available from: https://revistaepsi.com/artigo/ 2015-ano5-volume2-artigo2/

26. Padovani RC, Neufeld CB, Maltoni J, Barbosa LNF, Souza WF, Cavalcanti HAF, et al. Vulnerabilidade e bem-estar psicológicos do estudante universitário. Rev bras ter cogn. 2014;10(1):2-10. DOI: http:// dx.doi.org/10.5935/1808 5687.20140002

27. Souza MT, Silva MD, Carvalho R. Revisão integrativa: o que é e como fazer. Einstein 
(São Paulo). 2010;8(1):102-6. DOI: https://doi. org/10.1590/s1679-45082010rw1134

28. Sousa LMM, Marques-Vieira CMA, Severino SSP, Antunes AV. Metodologia de revisão integrativa da literatura em enfermagem. Rev Inv Enfer [Internet] 2017 [cited 2020 May 10];Ser II(21):7-26. Available from: https://repositorio-cientifico. essatla.pt/bitstream/20.500.12253/1311/1/ Metodologia\%20de\%20Revis\%c3\%a3o\%20 Integrativa_RIE21_17-26.pdf

29. Oliveira MAFD, Cestari TY, Pereira MO, Pinho PH, Gonçalves RMDDA, Claro HG. Processos de avaliação de serviços de saúde mental: uma revisão integrativa. Saúde debate. 2014;38(101):368-78. DOI: https://doi. org/10.5935/0103-1104.20140034

30. Santos CMC, Pimenta CAM, Nobre MRC. The PICO strategy for the research question construction and evidence search. Rev Latino-Am Enfermagem [Internet]. 2007 [cited 2020 Jun 10];15(3):508-11. Available from: http://www. scielo.br/pdf/rlae/ v15n3/v15n3a23.pdf

31. Stetler CB, Morsi D, Rucki S, Broughton S, Corrigan B, Fitzgerald J, et al. Utilization-focused integrative reviews in a nursing service. Appl Nurs Res. 2006;11(4):195-206. DOI: 10.1016/ s0897-1897(98)80329-7

32. Texeira MR, Dahl CM. Recriando cotidianos possíveis: construção de estratégias de apoio entre docentes e estudantes de graduação em Terapia Ocupacional em tempos de pandemia. Rev Interinst Bras Ter Ocup. 2020;4(3):509-18.

33. Yuan S, Liao Z, Huang H, Jiang B, Zhang X, Wang $\mathrm{Y}$, et al. Comparison of the Indicators of Psychological Stress in the Population of Hubei Province and Non-Endemic Provinces in China During Two Weeks During the Coronavirus Disease 2019 (COVID-19) Outbreak in February 2020. Med Sci Monit. 2020;26:e923767-1. DOI: 10.12659/ MSM.923767

34. Cao W, Fang Z, Hou G, Han M, Xu X, Dong J, et al. The psychological impact of the COVID-19 epidemic on college students in China. Psychiatry Res. 2020 May;287:112934. DOI: 10.1016/j. psychres.2020.112934

Recebido: 14 de junho de 2020

Aprovado: 31 de agosto de 2020

Publicado: 25 de novembro de 2020

A Revista Baiana de Enfermagem utiliza a Licença Creative Commons - Atribuição-NãoComercial 4.0 Internacional.

https://creativecommons.org/licenses/by-nc/4.0/

Este artigo é de acesso aberto distribuído sob os termos da Licença Creative Commons (CC BY-NC).

Esta licença permite que outros remixem, adaptem e criem a partir do seu trabalho para fins não comerciais. Embora os novos trabalhos tenham de lhe atribuir o devido crédito e não possam ser usados para fins comerciais, os usuários não têm de licenciar esses trabalhos derivados sob os mesmos termos. 